\title{
Questions and answers in EBD Volume 15
}

\author{
Derek Richards \\ Editor, Evidence-based Dentistry
}

As in previous years we are highlighting the guidelines, questions and answers addressed by summaries in Evidence-based Dentistry. Evidence levels are only given for those papers achieving level $3 \mathrm{~A}$ and above.

Evidence-Based Dentistry (2014) 15, 122-126. doi:10.1038/sj.ebd.6401068

\section{Guidelines}

\begin{tabular}{l|l}
\hline Topic & Page \\
\hline Topical Fluoride & $38-39$ \\
\hline Periodontal disease & $68-69$
\end{tabular}

\section{Summaries}

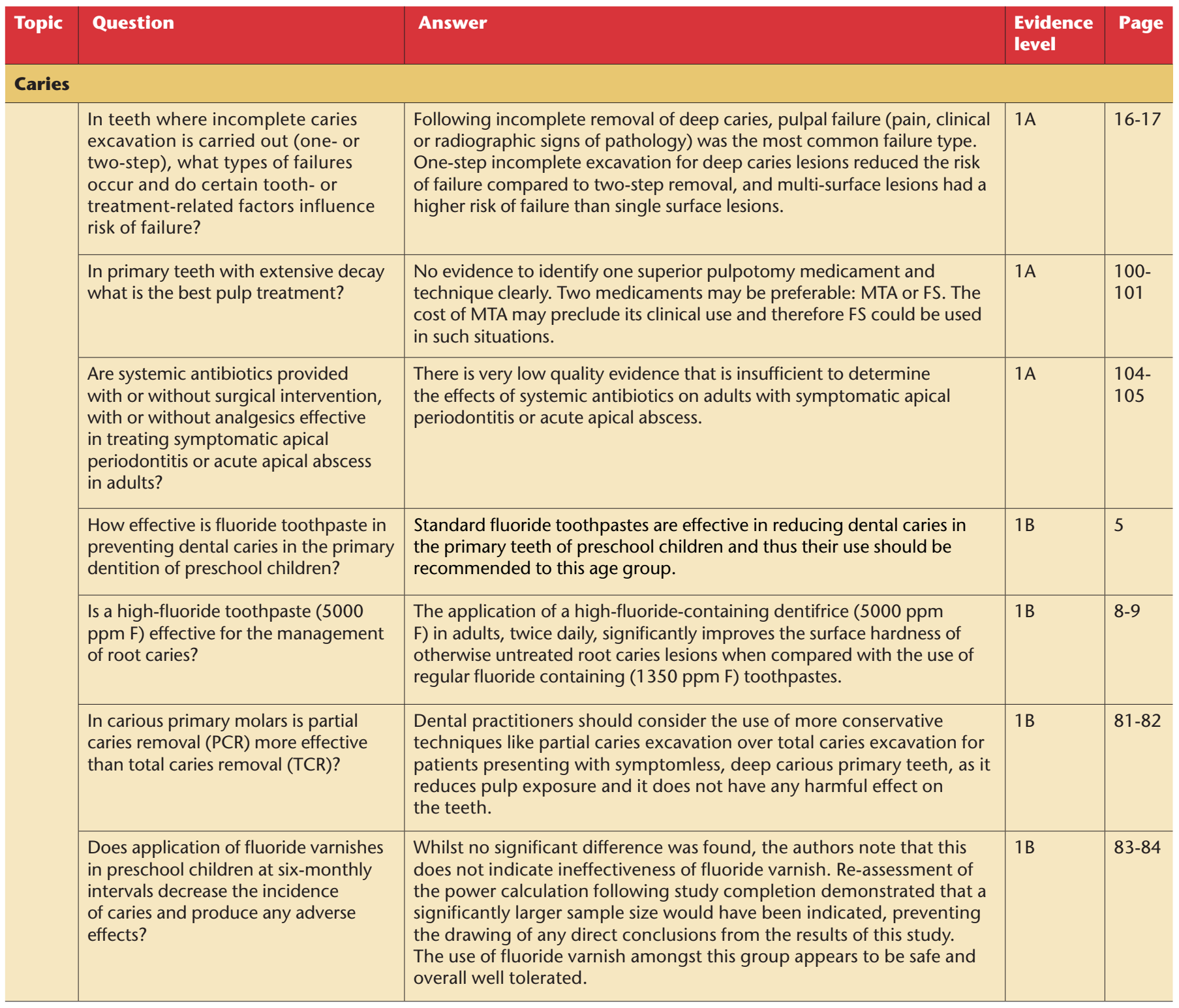


What is the effect of sugar consumption on dental caries? management of acute pulpitis?

Which caries prevention method for children is the most effective and economically viable?
Consistent evidence of moderate quality supporting a relationship between the amount of sugars consumed and dental caries development. There is evidence of moderate quality to show that dental caries is lower when freesugars intake is $<10 \% \mathrm{E}$. Dental caries progresses with age, and the effects of sugars on the dentition are lifelong. Even low levels of caries in childhood are of significance to levels of caries throughout the life course. Analysis of the data suggests that there may be benefit in limiting sugars to $<5 \% E$ to minimise the risk of dental caries throughout the life course.

In the light of an increased potential for antibiotic resistance and insufficient evidence to indicate the benefits of antibiotics in controlling pain due to irreversible pulpitis, dentists should desist from the practice of prescribing antibiotics for early irreversible pulpitis.

The non-operative caries treatment and prevention regimen was more effective and more costly than regular dental care. Based on the limited available amount of evidence regarding the willingness to pay for dental care, the benefits seem to outweigh the additional costs, implying that this is a cost-effective strategy. Increasing the number of professional fluoride applications resulted in a slight reduction of caries increment, but at higher costs than the NOCTP strategy. The results of this study confirm the findings in previous research. Therefore, implementing NOCTP on a larger scale should be considered.

\section{In children with approximal dentinal} caries lesions in primary molars do different treatment strategies affect behaviour and pain perception?

What is the prevalence of caries in children with cystic fibrosis (CF)?

Is there a difference in caries prevalence between cleft lip and palate (CLP) and a non-CLP population?

\section{More negative behaviours reported with conventional treatment} compared with Hall technique (HT) or non-restorative caries treatment (NRCT) with no local anaesthetic. with CF may not be at lower caries risk than those without CF. Additional research is needed to evaluate a potentially flawed paradigm regarding caries risk in children and adolescents with CF.

Individuals with CLP, when compared with matched non-CLP controls, tend to have a higher prevalence of dental caries, as detected using the decayed, missing and filled index. This holds true both for permanent and for deciduous teeth. One must keep in mind, however, that relatively few studies were included in the present meta-analysis, and the reporting on the methods in the individual studies was not always transparent.
While children with CF may be at lower risk for dental caries, adolescents

\section{Dental Implants}

Is periodontitis a risk factor for dental implant loss?

In patients with severely resorbed posterior ridges are short $(<10 \mathrm{~mm})$ implants with single crowns more effective than conventional implants and bone grafting?

Do implant overdentures improve the nutritional status of edentate elders?

Among patients receiving dental implants, does the use of antibiotics, when compared with a control group, reduce the frequency of implant failure and postoperative infection?

What is the influence of diabetes on dental implant failure rates?

What are the survival rates of sandblasted and acid-etched (SLA) dental implants and modified surface (SLActive) implants submitted to immediate or early occlusal loading?

Strong evidence suggests that periodontitis is a risk factor for implant loss; moderate evidence revealed that periodontitis is a risk factor for peri-implantitis and that patients with periodontitis have higher implant-bone loss.

Within the limitations of the present systematic review with metaanalysis, it is suggested that single crowns supported by short implants are an acceptable and predictable option in the short- and long-term treatment of the atrophic jaws.

Provision of implant-retained overdentures did not result in a significant improvement in dietary intake compared to conventional removable dentures.

The review concluded that the use of antibiotics significantly reduces implant failure but shows no superiority on postoperative infections.

$2 \mathrm{~A}$ 


\begin{tabular}{|l|}
\hline Does radiation therapy to the head, \\
for treatment of cancer, decrease the \\
survival rate of titanium dental implants \\
placed in non-grafted mouths?
\end{tabular}

Irradiation of the head was linked to increased failure rate of implants compared to failure rates in patients who had not undergone

radiotherapy. The failure rate was higher in the maxilla and HBO therapy did not improve implant survival.

\section{Endodontics}

Are electronic apex locators (EALs) accurate?

There is a high agreement rate between radiographs and EALs. When the two methods were compared, it is suggested that working length determination by using EAL may perform better than radiography alone.

\section{Effective practice and organisation of care (EPOC)}

Is using dental auxiliaries to provide care traditionally delivered by dentists effective?

What barriers to delivering oral health care to older people are experienced by dentists?

\section{Oral Health}

Are triclosan/copolymer containing fluoride toothpastes more effective than fluoride toothpastes for control of caries, plaque and gingivitis in children and adults?

Are powered toothbrushes as effective as manual toothbrushes in maintaining oral health?

Is motivational interviewing more effective than conventional education in changing oral health behaviours?

What is the optimum time period to have between one dental check-up and the next?
We only identified five studies for inclusion in this review, all of which were at high risk of bias, and four were published more than 20 years ago, highlighting the paucity of high-quality evaluations of the relative effectiveness, cost-effectiveness and safety of dental auxiliaries compared with dentists in performing clinical tasks. No firm conclusions could be drawn from the present review about the relative effectiveness of dental auxiliaries and dentists.

Lack of adequate dental equipment in a care home, concern for private practice and lack of adequate reimbursement were the most commonly reported barriers in the reviewed? publications. Lack of training and experience in providing oral care for this age group was also mentioned as a barrier.
The volume of moderate to high quality evidence supports that triclosan improves oral health outcomes.

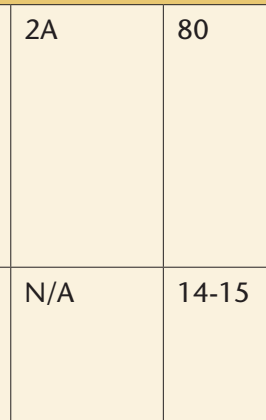

\section{Oral Medicine}

What are the effects of nonpharmacological interventions to stimulate saliva production for the relief of dry mouth?
Are antifungal treatments more effective than other therapies for the treatment of denture stomatitis?

Are prophylactic antibiotics required before invasive dental procedures in people at risk or at high risk of bacterial endocarditis?
There is low quality evidence that acupuncture is no different from placebo acupuncture with regard to dry mouth symptoms, which is the most important outcome. This may be because there were insufficient participants included in the two trials to show a possible effect, or it may be that there was some benefit due to 'placebo' acupuncture, which could have biased the effect to the null. There is insufficient evidence to determine the effects of electrostimulation devices on dry mouth symptoms. It is well known that dry mouth symptoms may be problematic even when saliva production is increased, yet only two of the trials that evaluated acupuncture reported dry mouth symptoms, a worrying reporting bias. There is some low quality evidence that acupuncture results in a small increase in saliva production in patients with dry mouth following radiotherapy. There is insufficient evidence to determine the effects of electrostimulation devices on dry mouth symptoms or saliva production in patients with Sjögren's Syndrome. Reported adverse effects of acupuncture are mild and of short duration, and there were no reported adverse effects from electrostimulation.

The findings of this review and meta-analysis suggest that disinfection methods could be considered as an adjunct or alternative to antifungal medications in the treatment of denture stomatitis.

There remains no evidence that antibiotic prophylaxis is either effective or ineffective against bacterial endocarditis in people considered at risk who are about to undergo an invasive dental procedure. It is not clear whether the potential harms and costs of penicillin administration outweigh any beneficial effect. Ethically, practitioners need to discuss the potential benefits and harms of antibiotic prophylaxis with their patients before a decision is made about administration. toothbrushing in the short and long term. The clinical importance of beneficial and harmful effects of different dental check-up recall intervals. There is no evidence to support or refute practice of six-monthly intervals.

\begin{tabular}{|l|l|l|}
\hline $\begin{array}{l}\text { The volume of moderate to high quality evidence supports that triclosan } \\
\text { improves oral health outcomes. }\end{array}$ & $1 \mathrm{~A}$ & $6-7$ \\
\hline $\begin{array}{l}\text { Powered toothbrushes reduce plaque and gingivitis more than manual } \\
\text { toothbrushing in the short and long term. The clinical importance of } \\
\text { these findings remains unclear. }\end{array}$ & $1 \mathrm{~A}$ & $77-78$ \\
\hline $\begin{array}{l}\text { Trials demonstrated variable success in improving oral health: current } \\
\text { evidence for benefit is inconclusive. }\end{array}$ & $2 \mathrm{~A}$ & $35-37$ \\
\hline $\begin{array}{l}\text { This was insufficient for drawing any conclusions about the potential } \\
\text { beneficial and harmful effects of different dental check-up recall intervals. } \\
\text { There is no evidence to support or refute practice of six-monthly intervals. }\end{array}$ & $2 \mathrm{~B}$ & 40 \\
\hline
\end{tabular}




\section{Oral Surgery}

Does tranexamic acid reduce blood loss during orthognathic surgery?

Following third molar removal which analgesic is most effective?

Should diseased teeth be extracted prior to radiotherapy to the jaw?

\section{Orthodontics}

What is the best orthodontic treatment approach for prominent upper front teeth?

What is the most effective treatment for class III malocclusion in children?

How effective are orthodontic appliances in distalising upper first molars in children and adolescents?

What is the best dental etchant and etching technique for bonding fixed orthodontic appliances?

Are surgical anchorage techniques more effective than conventional anchorage in preventing unwanted tooth movement during orthodontic treatment?

Are vacuum-formed retainers (VFRs) more effective than Hawley retainers (HRs) following orthodontic treatment?

In patients undergoing orthodontic treatment, can adjunctive interventions accelerate orthodontic tooth movement compared to conventional orthodontic treatment?

How do the characteristics of orthodontic brackets affect fixed appliance therapy?

What is the most effective archwire sequence for fixed appliance orthodontic treatment?

How effective are surgical and nonsurgical approaches for accelerated orthodontic tooth movement?
This meta-analysis confirms that tranexamic acid can effectively reduce intraoperative blood loss in orthognathic surgery, especially by intravenous administration. But tranexamic acid cannot affect postoperative levels of haemoglobin and haematocrit.

There is high quality evidence that ibuprofen is superior to paracetamol at doses of $200 \mathrm{mg}$ to $512 \mathrm{mg}$ and $600 \mathrm{mg}$ to $1000 \mathrm{mg}$ respectively based on pain relief and use of rescue medication data collected at six hours postoperatively. The majority of this evidence (five out of six trials) compared ibuprofen $400 \mathrm{mg}$ with paracetamol $1000 \mathrm{mg}$, these are the most frequently prescribed doses in clinical practice.

There are no randomised controlled trials to assess the effect of extracting teeth prior to radiotherapy compared to leaving teeth in the mouth during radiotherapy to the jaws. Current protocols based on evidence from quality observational studies for management of head and neck cancer patients provide comprehensive criteria for dental evaluation and treatment to be used for each case individually. None of the current practices recommends systematic extraction of healthy teeth.
The evidence suggests that providing early orthodontic treatment for children $\quad 1 \mathrm{~A}$ with prominent upper front teeth is more effective in reducing the incidence of incisal trauma than providing one course of orthodontic treatment when the child is in early adolescence. There appear to be no other advantages for providing treatment early when compared to treatment in adolescence.

There is some evidence that the use of a facemask to correct prominent lower front teeth in children is effective when compared to no treatment on a short-term basis. However, in view of the general poor quality of the included studies, these results should be viewed with caution. Further randomised controlled trials with long follow-up are required.

It is suggested that intraoral appliances are more effective than headgear in distalising upper first molars. However, this effect is counteracted by loss of anterior anchorage, which was not found to occur with headgear when compared with intraoral distalising appliance in a small number of studies. The number of trials assessing the effects of orthodontic treatment for distilisation is low, and the current evidence is of low or very low quality.

There is limited evidence to suggest that no difference exists between orthodontic attachment failure rates with the acid-etch technique and self-etch primers.

There is moderate quality evidence that reinforcement of anchorage is more effective with surgical anchorage than conventional anchorage, and that results from mini-screw implants are particularly promising. While surgical anchorage is not associated with the inherent risks and compliance issues related to extra-oral headgear, none of the included studies reported on harms of surgical or conventional anchorage.

This systematic review suggests that further high-quality RCTs regarding the differences between HRs and VFRs during orthodontic retention are necessary to determine which retainer is the better selection for orthodontists.

Limited evidence suggests that corticotomy may safely speed up the $1 \mathrm{~A}$ orthodontic tooth movement; however, its cost-effectiveness needs to be further assessed. Evidence is inconclusive for the effectiveness of other interventional techniques.

There appears to be no advantage in the use of self-ligating brackets when compared to conventional brackets for orthodontic treatment.

\begin{tabular}{|l|l|}
\hline $1 \mathrm{~A}$ & 63 \\
\hline $1 \mathrm{~A}$ & $\begin{array}{l}106- \\
107\end{array}$ \\
\hline N/A & 76 \\
\end{tabular}

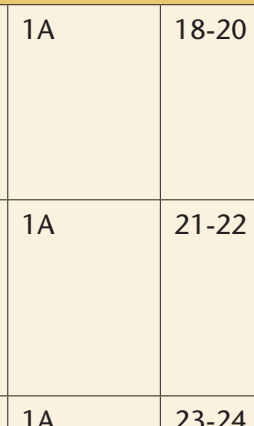

The available evidence is limited to produce strongly supported conclusions, but it seems that there are no differences among NiTi alloys to successfully complete orthodontic levelling and alignment. Other factors should be taken into account when selecting from the commercially available options.

There is some evidence that low intensity laser therapy and corticotomy are effective, whereas the evidence is weak for interseptal bone reduction and very weak for photobiomodulation and pulsed electromagnetic fields. Overall, the results should be interpreted with caution given the small number of studies, allied to limited quality and heterogeneity of the included studies. 


\section{Paediatric dentistry}

What are the characteristics of child dental neglect?

This systematic review clarifies the key signs to trigger consideration of dental neglect: failure to seek or a delay in seeking dental treatment; failure to follow dental advice; failure to administer medicine and provide basic oral care, more importantly it high- lights what still needs to be done.

\section{Periodontal disease}

Is routine scale and polish beneficial for oral health?

Does non-surgical periodontal treatment improve glycaemic control in diabetic patients?

Is there an association between smoking and periodontitis?

What is the global prevalence and incidence of severe periodontitis (SP)?

\begin{tabular}{|l|l|l}
\hline $\begin{array}{l}\text { There is insufficient evidence to determine the effects of routine scale and } \\
\text { polish treatments. }\end{array}$ & $1 \mathrm{~A}$ & $74-75$ \\
\hline $\begin{array}{l}\text { While this review suggested a small benefit to glycaemic control, whether } \\
\text { the improvement is clinically significant is questionable. }\end{array}$ & $1 \mathrm{~A}$ & $93-94$ \\
\hline $\begin{array}{l}\text { This research confirmed the strong association between chronic smoking } \\
\text { and periodontal disease. }\end{array}$ & $1 \mathrm{~B}$ & $72-73$ \\
\hline \begin{tabular}{l} 
Severe periodontitis affects 11\% of the world's population. \\
\hline
\end{tabular} & $2 \mathrm{~A}$ & $70-71$ \\
\hline
\end{tabular}

\section{Restorative Dentistry}

What factors affect the survival of posterior composite restorations?

What is the failure rate of direct composite resin fillings versus amalgam fillings for permanent posterior teeth?

Is basic root canal treatment (BRT) with tactile working length determination as effective as standard endodontic treatment after 24 months?

For patients with ameliogenesis imperfect (Al) what are the most effective restorative materials and techniques?

Should we repair or replace defective composite restorations?

Should we repair or replace defective amalgam restorations?
The conclusion of the present meta-analysis of 12 clinical studies based on raw data is that caries risk and number of restored surfaces play a significant role in restoration survival, and that, on average, posterior resin composite restorations show a good survival, with annual failure rates of $1.8 \%$ at five years and $2.4 \%$ after ten years of service.

There is low-quality evidence to suggest that resin composites lead to higher failure rates and risk of secondary caries than amalgam restorations.

Within the limits of this randomised controlled trial it was shown that tactile working length determination in BRT resulted in comparable treatment outcomes in radiographic and clinical aspects compared with standard endodontic treatment with radiographic working length determination. Tactile working length determination turned out to be an accurate method in BRT.

There are no randomised controlled trials of restorative treatments for children and adolescents with $\mathrm{Al}$, and therefore there is no evidence as to which is the best restoration. Well defined randomised controlled trials which recruit children and adolescents and focus on the type and severity of the disorder should be undertaken to determine the best intervention for restoring teeth affected by Al.

There are no published randomised controlled trials relevant to this review question. Therefore there is a need for methodologically sound randomised controlled trials that are reported according to the Consolidated Standards of Reporting Trials (CONSORT) statement (www. consort-statement.org/

There are no published randomised controlled trials relevant to this review question. Therefore there is a need for methodologically sound randomised controlled trials that are reported according to the Consolidated Standards of Reporting Trials (CONSORT) statement (www. consort-statement.org/

\begin{tabular}{l|l|l}
\hline IA & $\begin{array}{l}118- \\
119\end{array}$ \\
\hline $2 A$ & $50-51$ \\
\hline & $2 B$ & $56-57$
\end{tabular}

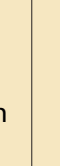

\section{Temporomandibular disorders}

In patients with temporomandibular joint (TMJ) disc displacement without reduction (DDwoR) are conservative or surgical interventions more effective?
Most interventions appear to alleviate DDwoR symptoms, with no significant differences between non-invasive conservative interventions and minimally invasive or invasive surgical interventions. Given the paucity of evidence and the difficulty in interpreting the minimal clinically important difference, this finding suggests that patients with DDwoR probably should be initially managed with the most minimal and least invasive intervention.

$3 A$

$2-4$ $2-4$ 